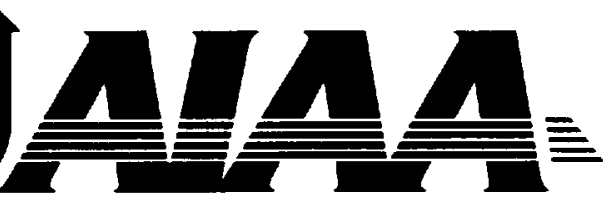

AIAA-95-3463

Unsteady Simulation of the Viscous Flow About a V-22 Rotor and Wing in Hover

\title{
R. Meakin
}

Overset Methods, Inc.

Moffett Field, CA

\section{AIAA Atmospheric Flight Mechanics Conference August 7-10, 1995 / Baltimore, MD}




\title{
Unsteady Simulation of the Viscous Flow About a V-22 Rotor and Wing in Hover
}

\author{
Robert L. Meakin $†$ \\ Overset Methods, Inc. \\ at NASA Ames Research Center M/S 258-1 \\ Moffett Field, CA 94035
}

\begin{abstract}
Results of an unsteady thin-layer Navier-Stokes simulation of a 0.658 -scale V-22 rotor and wing configuration in hover are presented. All geometric components of the flapped-wing and rotor test rig, including rotor blades, are accurately modeled. Rotor motion and rotor/ airframe interference effects are simulated directly using moving body overset grid methods. Tiltrotor hover aerodynamics are visualized via unsteady particle trace images. Wing download predictive ability is demonstrated. Simulation results are compared with experimental data.
\end{abstract}

\section{INTRODUCTION}

One of the most important factors in the overall utility of a tiltrotor aircraft is its aerodynamic performance in hover. Payload capacity is determined by performance in hover and is directly related to the potential cost effectiveness of the aircraft for either civil or military applications. Noise is a key issue for the environmental acceptability of future civil tiltrotor aircraft. It is in hover conditions, such as take-off and landing maneuvers, where noise is an issue. An understanding of hover aerodynamics is very important to the design of efficient and environmentally acceptable tiltrotor aircraft.

The flowfield about a tiltrotor aircraft in hover is very complex. Blade-tip vortices are relatively high frequency flow structures which convect down and impinge on the wings. The tilt-rotor wakes interact with the wings to form a lower frequency recirculating flow condition over the fuselage/wing junction known as the tiltrotor "fountain." The rotor downwash over the wings results in massive regions of separated flow on and below the wing. Blade interaction with tip-vortices and the fountain are primary sources of noise.

An unsteady Navier-Stokes simulation of a 0.658 scale V-22 rotor and flapped-wing configuration has been carried out to study the aerodynamics of a tiltrotor in hover. Of particular interest is the mechanism of for- mation and dynamics of the tiltrotor fountain. The simulation conditions were derived from the experiments of Felker, et al. ${ }^{\prime}$ carried out in the NASA Ames $40 \times 80$ foot wind tunnel. The simulation includes accurate modeling of all geometric components of the flapped-wing and rotor test rig, including rotor blades. Accordingly, rotor motion and rotor/airframe interference effects are directly simulated. The simulation also represents a test case of a general purpose flow solver that has recently been unified with an efficient algorithm for establishing domain connectivity among systems of overset grids.

Results of the simulation are presented in the following pages. The paper is organized into three main sections. First, the specific test configuration studied is defined in terms of geometry and flow parameters. Differences/ambiguities between the experiment and simulation configurations are noted. Second, the numerical methods employed for the simulation are described briefly and appropriate references to the literature are given. Evidence of the formal accuracy of the numerical methods is also provided. Finally, a thorough analysis of the simulation results is given. Comparison with experimental results is made whenever possible. Simulation results are presented graphically to illustrate the qualitative characteristics of all key flow structures.

\section{TEST CONFIGURATION DEFINITION}

The basic test configuration used for Felker's experiment and the present simulation is indicated in Figure 1. Inspection of this figure reveals several presumably minor differences between the two representations of the test configuration. The experimental article was set up in the $40 \times 80$ foot wind tunnel in a configuration intended to represent free-air conditions to the degree possible in an indoor facility. The test section overhead doors were left open during the tests and the rotor wake was directed into a large settling chamber and outside through an exhaust path. The CFD simulation corresponds exactly to free air conditions. No effort was made to account for the tunnel walls, overhead doors, etc. in the computation.

\footnotetext{
†Staff Scientist, Member AIAA

Copyright $(1) 1995$ by Robert L. Meakin. Published by the American Institute of Aeronautics and Astronautics, Inc. with permission.
} 
for the actual V-22 rotor. The rotor blade definition used in the present simulation corresponds to the actual V-22 rotor. The wing sweep and dihedral angles, and rotor cant angle used in the present computational model correspond to the experimental values indicated in Figure 3.

Table 1: Basic Dimensions

\begin{tabular}{|c|c|c|}
\hline $\mathrm{R}$ & rotor radius & $12.5 \mathrm{ft}$ \\
\hline $\mathrm{R}_{\mathrm{n}}$ & nacelle radius & $1.67 \mathrm{ft}$ \\
\hline
\end{tabular}

Reference 1 contains experimental results for a variety of flow conditions, exploring the effects of flap angle, nacelle angle, rotor thrust, blade-tip speed, and sense of rotation on tiltrotor wing downloads. The present computational study corresponds to the Run 6 Point 9 case described in Reference 1. The case parameters are summarized below in Table 2 .

Table 2: Simulation Case Definition

\begin{tabular}{|c|c|c|}
\hline$\alpha_{b}$ & blade collective pitch angle & $12^{\circ}$ \\
\hline$\alpha_{\mathrm{f}}$ & flap angle & $67^{\circ}$ \\
\hline$\alpha_{\mathrm{n}}$ & nacelle angle & $85^{\circ}$ \\
\hline$S_{\mathrm{r}}$ & $\begin{array}{c}\text { sense of rotor rotation } \\
(\mathrm{w} / \mathrm{r} \text { pilot fwd, right wing })\end{array}$ & $\begin{array}{c}\text { right } \\
\text { handed }\end{array}$ \\
\hline$v_{\text {tip }}$ & blade-tip speed & $803.7 \mathrm{ft} / \mathrm{s}$ \\
\hline
\end{tabular}

The blade collective pitch angle $\alpha_{b}$ was not specified in Reference 1. Rather, the case was defined by the rotor thrust $\left(C_{T}\right)$ and power $\left(C_{Q}\right)$ coefficients. Rotor thrust and shaft torque (i.e. power) are functions of blade pitch angle. Accordingly, the blade pitch angle used in the simulation was set to produce the experimentally measured thrust and power (see Table 3 ).

Table 3: Thrust and Power Coefficients

\begin{tabular}{|c|c|c|}
\hline & $\mathrm{C}_{\mathbf{T}}$ & $\mathrm{C}_{\mathrm{Q}}$ \\
\hline Experiment $^{\prime}$ & 0.01476 & 0.001568 \\
\hline Present Simulation & 0.01419 & 0.001565 \\
\hline
\end{tabular}

The present computational study was carried out using dimensionless variables. All results presented in subsequent sections of the paper will be in dimensionless form. Accordingly, for completeness, it is necessary to define the characteristic variables used in the nondimensionalization process. The characteristic variables and other key variables are defined in Table 4.

Table 4: Characteristic Variables, Units, and Definitions

\begin{tabular}{|c|c|c|}
\hline$L$ & rotor radius, $R$ & $12.5 \mathrm{ft}$ \\
\hline$V$ & sonic speed & $1121 \mathrm{ft} / \mathrm{s}$ \\
\hline$\rho$ & air mass density & $0.002355 \mathrm{lb}-\mathrm{ft} / \mathrm{s}$ \\
\hline$\mu$ & air dynamic viscosity & $3.719 \times 10^{-7} \mathrm{lb}-\mathrm{s} / \mathrm{ft}^{2}$ \\
\hline$C_{\mathrm{Dl}}$ & wing download coef. & $\mathrm{Dl} / \rho \mathrm{A}(\Omega \mathrm{R})^{2}$ \\
\hline $\mathrm{C}_{\mathrm{p}}$ & pressure coef. & $(\mathrm{P}-\mathrm{P} \mathrm{inf}) /(\mathrm{T} / \mathrm{A})$ \\
\hline $\mathrm{C}_{\mathrm{Q}}$ & rotor power coef. & $\mathrm{Q} \Omega / \rho \mathrm{A}(\Omega \mathrm{R})^{3}$ \\
\hline $\mathrm{C}_{\mathrm{T}}$ & rotor thrust coef. & $\mathrm{T} / \rho \mathrm{A}(\Omega \mathrm{R})^{2}$ \\
\hline $\mathrm{A}$ & rotor disc area & $\pi \mathrm{R}^{2}$ \\
\hline $\mathrm{Dl}$ & wing down load & $\mathrm{lb}$ \\
\hline $\mathrm{P}_{\mathrm{inf}}$ & free-stream pressure & $\mathrm{lb} / \mathrm{ft}^{2}$ \\
\hline $\mathrm{Q}$ & rotor shaft torque & $\mathrm{ft}-\mathrm{lb}$ \\
\hline $\mathrm{T}$ & (power = Q $\Omega$ ) & $\mathrm{lb}$ \\
\hline$\Omega$ & rotor angular velocity & $\mathrm{v}_{\mathrm{tip}} / \mathrm{R}$ \\
\hline
\end{tabular}

\section{NUMERICAL METHODS}

The present test case poses a number of problems from a computational point of view. In addition to aerodynamic complexities, the test configuration is complicated by formidable geometric obstacles as well. The geometry is complex and involves relative motion between component parts (i.e., blade motion $w / r$ rotor test rig and flapped-wing). The Chimera overset grid method $^{2}$ of domain decomposition has been used to discretize the configuration geometry and form the basis of the computational effort.

An overset grid approach offers many advantages for this type of problem. The geometric complexity of the configuration can be reduced to a number of geometrically simple component parts and discretized via corresponding overlapping structured grid systems. Accordingly, grid generation is greatly simplified relative to other approaches. Grid components can move independently with six degrees of freedom relative to an inertial frame of reference. As a result, blade/vortex and rotor/wing interaction can be directly simulated without 
than near the blades, was assumed to be laminar. Clearly, this is not a correct assumption. However, it is one necessitated by expedience because of the lack of an appropriate model of turbulence for the rotor wake, wake impingement on the wing, and massively separated flow under the wing.

Table 5 summarizes flow parameter settings used in the flow solver for the present tiltrotor simulation. The Reynolds number given in Table 5 is based on $v_{\text {tip }}$ to conform with the convention common to the rotorcraft literature. However, the flow solver divides this value by $\mathbf{M}_{\text {tip }}$ to maintain its internal non-dimensionalization, which uses the sonic speed, rather than the blade-tip speed, as the characteristic velocity. Noting the values of $\Delta \mathrm{t}$ and $\mathrm{t}_{\text {rev }}$ given in Table 5 , it is apparent that more than 3,500 time-steps were used for each revolution of the rotor. This translates to approximately $0.1^{\circ}$ of blade rotation per time-step.

Table 5: Simulation Parameters

\begin{tabular}{|c|c|c|}
\hline $\mathrm{M}_{\text {tip }}$ & blade-tip Mach number & 0.717 \\
\hline $\mathrm{M}_{\mathrm{inf}}$ & free-stream Mach number & 0 \\
\hline $\mathrm{R}_{\mathrm{e}}$ & $\begin{array}{c}\text { Reynolds number } \\
\text { (based on } \mathrm{v}_{\mathrm{tip}} \text { ) }\end{array}$ & $63.6 \times 10^{6}$ \\
\hline$\Delta \mathrm{t}$ & dimensionless time-step & 0.0025 \\
\hline $\mathrm{t}_{\mathrm{rev}}$ & $\begin{array}{c}\text { dimensionless time for } 1 \\
\text { revolution of the rotor }\end{array}$ & 8.76 \\
\hline
\end{tabular}

\section{Solver Accuracy}

The formal accuracy of the flow solver is second order in space and, optionally, first, or second order in time. Since the Euler implicit time integration option was used in the present work, the temporal accuracy was first order. The formal temporal and spatial accuracy of the solver has been demonstrated previously on overlapping grids via grid refinement studies. ${ }^{13}$ Figure 5 illustrates the rate of spatial error reduction that was realized in a grid refinement study using overlapping curvilinear grids (2D).

Formal accuracy only indicates the rate at which the difference equations converge to the governing differential equations as grid spacing is reduced. The absolute accuracy of the solution to a practical application depends on how well the evolving aerodynamic fields have been resolved by the grids employed. Lacking resources to carry out grid refinement studies to demonstrate grid independence, it is not possible to make

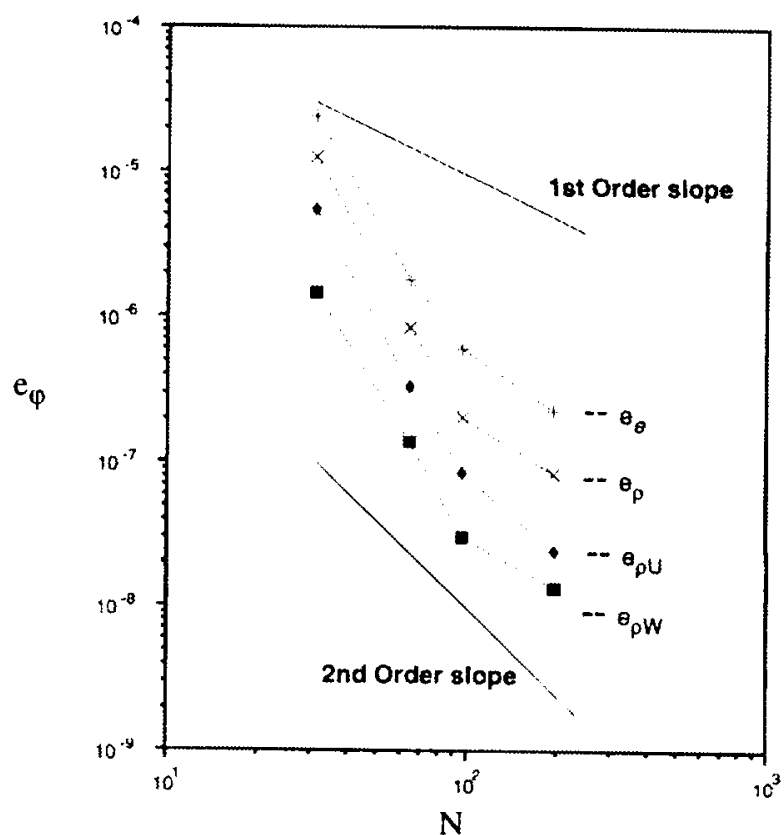

$e_{\varphi}=\frac{1}{N} \sum_{i=1}^{N}\left|\varphi_{\text {overset }}-\varphi_{\text {ref }}\right|_{i}$

Figure 5. Average absolute error reduction with increasing grid resolution for $2 \mathrm{D}$ overset grid case.

definitive statements as to the accuracy of the present tiltrotor solution. Accordingly, qualitative assessments of accuracy and comparison with available data to verify physical consistency will be done in this case.

The temporal resolution used in the present simulation was excellent ( $>3,500$ steps per rev). The spatial resolution of the configuration's geometric surfaces is also very good. However, due to the complexity of the tiltrotor aerodynamic field, and limited computational resources, the spatial resolution of the off-body field is good, but not optimal.

\section{Grid Topology}

The approach for geometry decomposition and domain discretization was to use a combination of background Cartesian grids and topologically simple bodyfitted grids. The background Cartesian grids are a simple way to efficiently discretize the overall domain and resolve the dominant flow features away from the rotor and wing. The body-fitted grids resolve the geometric detail of the configuration, and the viscous dominated regions of the surrounding flowfield. In all, 26 component grids were used, totalling more than 2.5 million grid points.

The background Cartesian system of grids consists of 
The body-fitted grid components have been limited to two simple topologies; "cap" and "O" grids. "C" grids and topologies that require special axis logic were avoided because, in the author's view, they are not well suited to unsteady flow problems. Although " $\mathrm{C}$ " topologies are very common, they are prone to inappropriate use within an overset grid approach. There are two main reasons for this. First, the viscous spacing which is required for boundary layer resolution is propagated along the C-slit to the grid outer boundary. Therefore, solutions interpolated from neighboring grid systems will likely be obtained from a grid of vastly different resolution capacity, possibly resulting in decreased solution accuracy. Second, a "C" topology often assumes wake location to be coincident with the C-slit, which is only the case for simple ideal problems. The wake location is often unsteady, and is usually influenced by interference effects from other body components. The approach recommended here is to choose topologically simple body-fitted grids that have surface geometry resolution as their prime objective. The role of unsteady flow dynamics resolution belongs to adaptive grid techniques which can be implemented via solution adaptive overset gridding. ${ }^{14}$

Topologies that require axis logic are undesirable for different reasons. Axis logic generally implies that the solution along the axis is derived from an averaging process involving a nearby off-axis solution. If grid clustering is sufficiently fine near the axis, such topologies can be effective. However, it is usually impractical to provide the needed axis clustering, and the numerical stability constraints imposed by their use are often severe.

In an attempt to enhance resolution of off-body dynamics of the blade-tip vortices, a very fine background grid system was generated using the topology of a cylindrical annulus. Clearly, given a case involving an isolated rotor in hover, one could usefully generate and a-priori position such a grid since the gross rotor wake behavior would be known. However, in the present case, the wing interacts with the rotor wake and, therefore, it is not clear that such a fine grid will actually improve the resolution of the blade-tip vortices. The fine grid shown in Figure 10 was used in the simulation. The added grid points offer a real potential for more accuracy, with no risk of accuracy degradation.

The overset grid system employed for the present tiltrotor case has a total of 26 component grids, and more than 2.5 million grid points in all. The domain connectivity requirements of the problem vary from one timestep to the next due to the motion of the rotor blades. However, connectivity requirements for intergrid

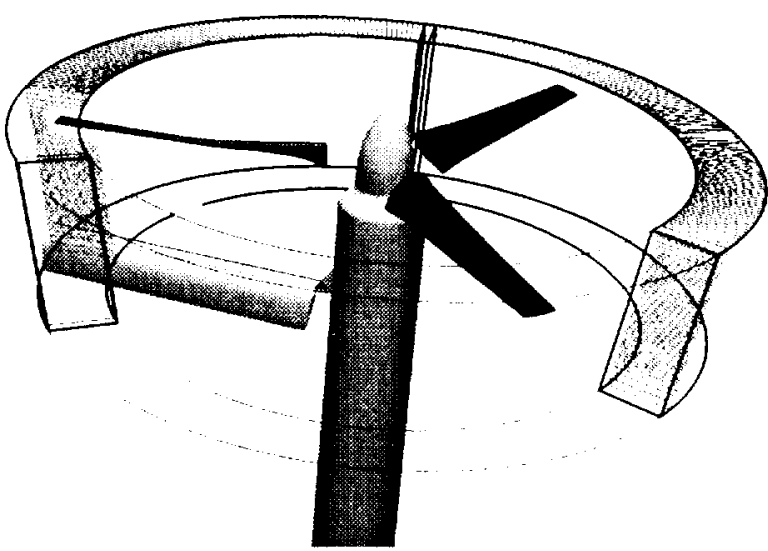

Figure 10. Fine background grid to help resolve offbody dynamics of blade-tip vortices.

boundary points (IGBPs) unaffected by blade motion are not recomputed, interpolation information available at time-level " $n$ " is reused at time-level " $n+1$ ". The average domain connectivity requirements of all types for the present case are 265,000 IGBPs/time-step. On a CRAY Y/MP C-90, the domain connectivity requirements were satisfied at a rate of approximately 10,000 IGBPs per CPU second.

\section{SIMULATION ANALYSIS}

\section{Flowfield Characteristics}

The tiltrotor test case described previously was started impulsively from quiescent flow conditions and run time-accurately for 17 revolutions. Histograms of rotor thrust and shaft-torque (power), and wing download are shown in Figure 11. Perhaps the first point that should be noted about the histograms is that they indicate that the simulation has not been carried out far enough to prove that a periodic state has been reached. Although the mean rotor thrust and power values seem to have settled down, their signatures are still changing as indicated by slowly increasing amplitude and irregular high frequency blips associated with each blade event. The wing download mean value appears to be leveling out.

As a means of discussion of the simulation results, consider the three major periods indicated below the time axis in Figure 11: rotor start-up, fountain formation, and rotor/wing/fountain interaction. The start-up period is characterized by interaction between a start-up ring-vortex and the out-board blade-tip vortices (see Figure 12a). The blade-tip vortices are well defined structures, but are eventually entrained by the start-up ring vortex, adding to the strength of its vorticity. Note that for a right-handed wing and rotor, the sense of vor- 


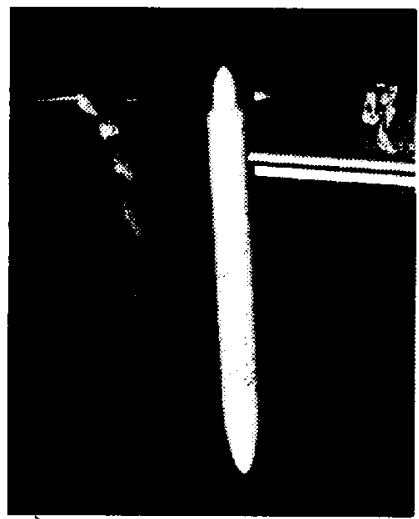

a)

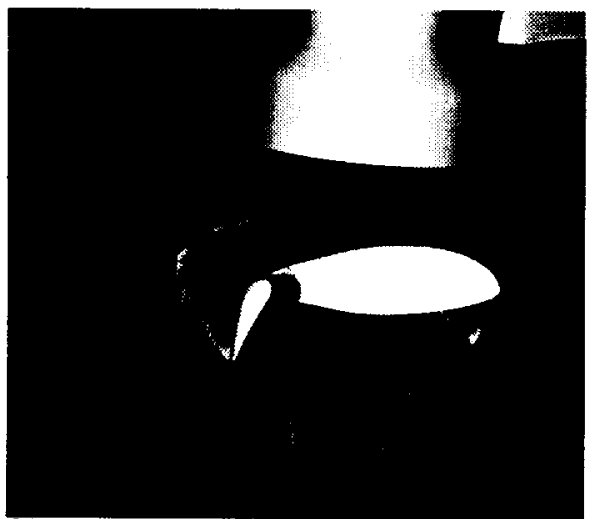

b)

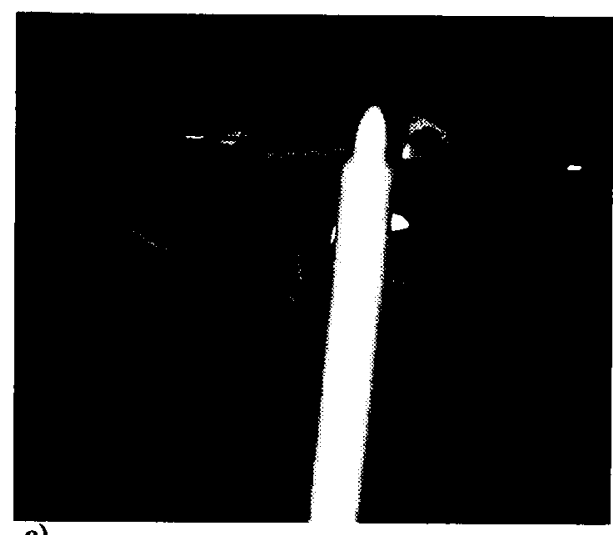

c)

Figure 12. Start-up. Particles are released from seed locations at specified time intervals and allowed to convect with the unsteady flow field (images taken at $t=42.625$ ). a) Front view: particles released along a line in the plane of the rotor every $50 \Delta t$. b) Side view (nacelle made invisible): particles released once per rev from a matrix of seed points located just above the wing and flap upper surfaces. c) Side view: particles released once per rev from a square matrix of seed points in the symmetry plane (particle motion restricted to symmetry plane). Note: particle path images were created in a post-process using UFAT..$^{15}$

symmetry plane and above the wing upper surface. Figure $12 \mathrm{c}$ illustrates the flow direction in the plane of symmetry. Particles have been released once per rev from a square matrix of seed points in the plane of symmetry. The rotor has been running for $\sim 5$ revs. Particles were released once per rev for the last 3 revs in Figures 12b and $12 \mathrm{c}$. At this stage of the flow, the effect of the rotor is to entrain air between the symmetry plane and the rotor disk, creating a downward flow toward the wing. This downwash is in opposition to the radial flow away from the wing near the plane of symmetry. The ultimate strength and height of the tiltrotor fountain represents the equilibrium state of the opposing forces of the rotor downwash and wing/symmetry plane radial outflow.

As the rotor wake begins to establish itself, the rest of the flow structures around the rotor and wing likewise continue to evolve. Figure 13 illustrates the flow characteristics after $\sim 10$ revs $(t=86.375)$. Comparison of Figure 13a with Figure 12a shows that the rotor wake has increased in extent and intensity. The wake appears to be entraining more air and the rotor wake shear-layer has overpowered the tip-vortices. The tip vortices maintain their structure to a greater extent in reality. ${ }^{16}$ However, in the simulation, the tip vortex intensities are under predicted due to insufficient grid resolution.

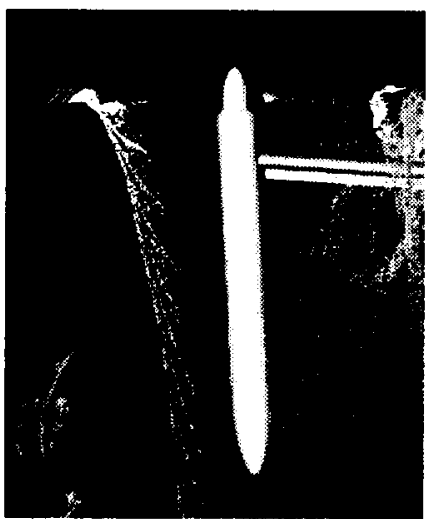

a)

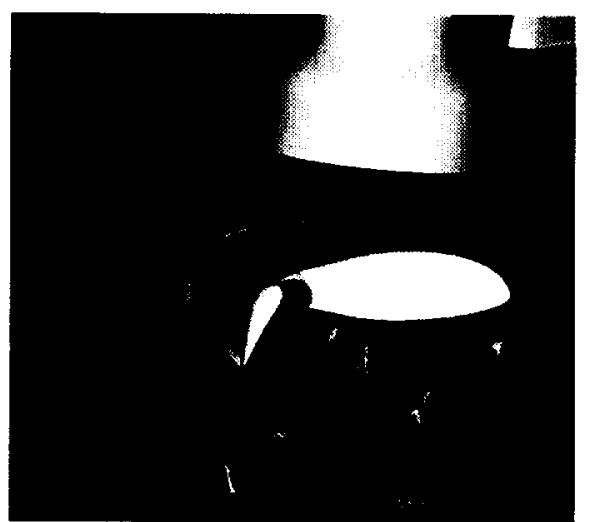

b)

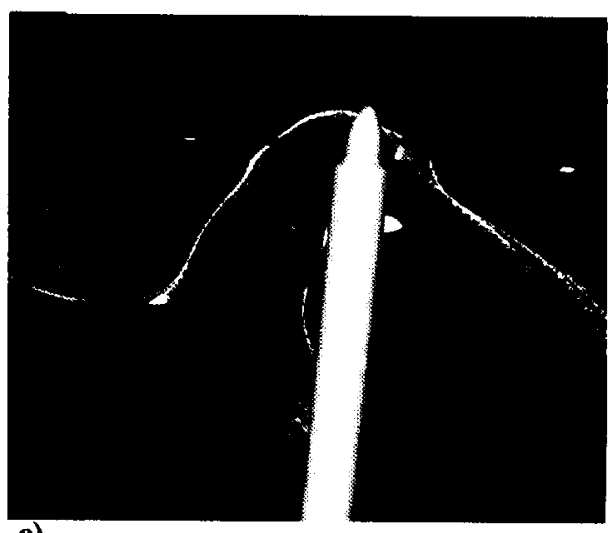

c)

Figure 13. Fountain formulation. Particles are released from seed locations at specified time intervals and allowed to convect with the unsteady flow field (images taken at $t=86.375$ ). a) Front view: particles released along a line in the plane of the rotor every $50 \Delta \mathrm{t}$. b) Side view (nacelle made invisible): particles released once per rev from a matrix of seed points located just above the wing and flap upper surfaces. c) Side view: particles released once per rev from a square matrix of seed points in the symmetry plane. 
The primary questions that remain after 17 revs of the simulation concern the equilibrium state of the flowfield. Has a periodic state been reached, or is there a low frequency oscillation that still needs to settle out? The significance of this question is two-fold. First, the peak and mean wing surface pressures and integral loads depend on the temporal state of the flowfield. These values are controlled by the period associated with the lowest significant frequency of oscillation. Second, from a practical standpoint, the existence of significant low frequency oscillations directly controls the computational expense of a numerical simulation (i.e., lower frequency equals higher computational expense).

\section{Comparison with Experimental Data}

Comparisons between the present simulation and experimental data have been carried out. The sampling period used in the experiment to determine mean quantities was not specified in Reference 1. In the present paper, mean quantities from the simulation are based on 1 -rev simple averages.

Comparison between the computed and experimentally measured mean rotor thrust and shaft torque coefficients are given in Table 3 . The computed values of $\mathrm{C}_{\mathrm{T}}$ and $\mathrm{C}_{\mathrm{Q}}$ are less than $4 \%$ and $1 \%$ of the respective values measured in the experiment. Figure 15 shows the wing and flap upper surfaces grey-scale shaded by mean $C_{p}$. The portion of the wing and flap surfaces shaded by $C_{p}$ correspond to the wing and flap sections instrumented in the experiment. Also, sectional location of pressure-tap rows in the experiment are indicated by black lines in the figure. Figure 16 compares computed and experi- mental mean $C_{p}$ distributions over the upper and lower surfaces of the wing and flap.

Although the comparisons between experimental mean $C_{p}$ distributions and the 1 -rev averages from the simulation are in general agreement, there are some differences that should be noted. Near the nacelle, the agreement between experiment and simulation is excellent. However, the agreement at stations 0.85 and 1.05 . is less favorable. The area between these two stations is where the tip-vortices impinge directly on the wing and flap upper surfaces. The area of the wing for $r / R$ greater than 0.85 also corresponds to the region of flow that has undergone large changes associated with the formation of the fountain, and may still be in a state of flux.

The 1-rev mean wing download $\left(\mathrm{C}_{\mathrm{DI}}\right)$ that results from the simulation (17th rev) is 0.00137 , which is $8 \%$ higher than the value of 0.00127 reported in Reference 1. The computational and experimental values of $C_{D I}$ were both obtained by integration of pressure over the wing and flap surfaces.

The present agreement between computed and experimental download for this case is somewhat remarkable. Previous independent computational results for this case have overpredicted the reported value of experimental wing download by more than 33 percent. ${ }^{17}$ The fidelity of the present simulation with experimental data, relative to previous results, can be explained largely by the fidelity of the present discretization with the actual test article. The present simulation used approximately 10 times the number of grid-points (with essentially no increase in memory requirements) and 25 times the

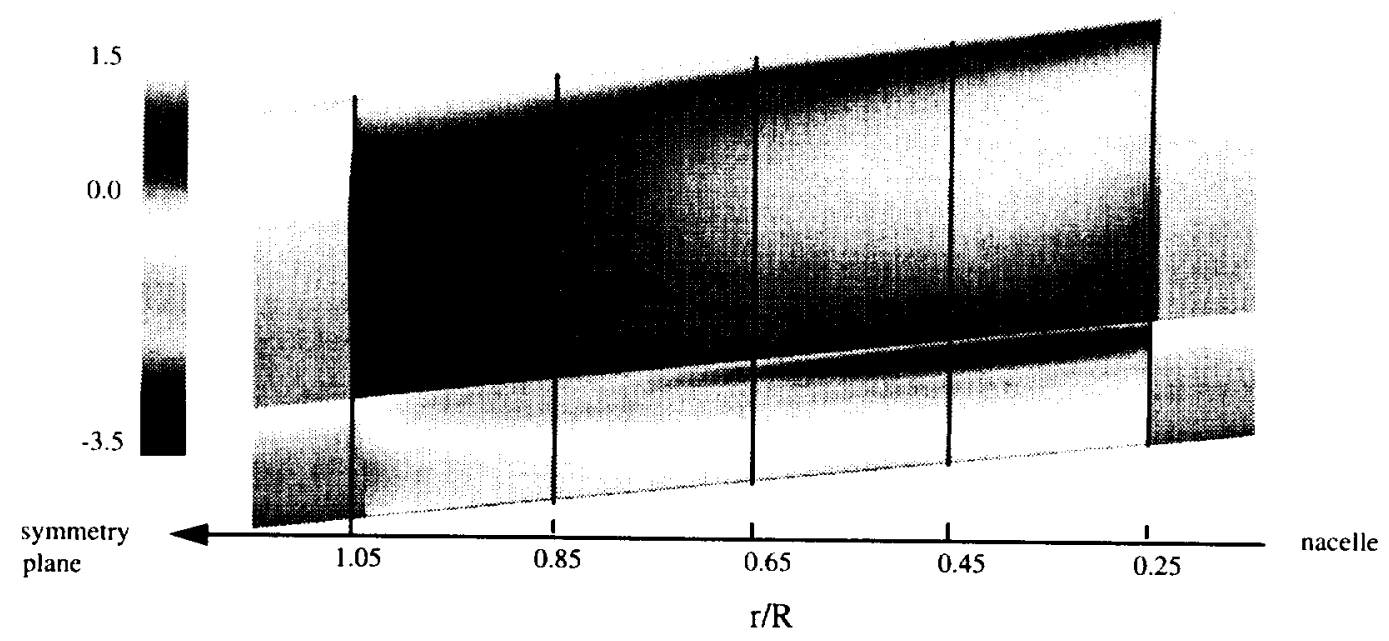

Figure 15. Computed $\mathrm{Cp}$ distribution on the wing and flap upper surfaces (1-rev averaged values of rev 17). 
enough to know for sure, or to ascertain whether other low frequency oscillatory flow features exist. Finally, an appropriate model of turbulence does not exist for this flowfield.

Future research in the computational aspects of this problem will be directed toward the development and testing of solution adaption techniques for overset grid systems. This will facilitate higher fidelity solutions while efficiently using available computational resources. Given an overset grid based solution adaptive capability, the aerodynamics of tiltrotor aircraft in hover will be studied further, with emphasis on issues of acoustics and download prediction.

\section{ACKNOWLEDGEMENTS}

This work was carried out under NASA ARC grant NCC2-747. All computational results reported herein were carried out on the NAS facility at ARC. This support is gratefully acknowledged. The author wishes to acknowledge the influence of Dr. Jim McCroskey who has encouraged this work and provided many insights. The author is also indebted to Bell Helicopter Textron, Inc. and Boeing Helicopters for their interest in the project.

\section{REFERENCES}

${ }^{1}$ Felker, F., Shinoda, P., Heffernan, R., and Sheehy, F., "Wing Force and Surface Pressure Data from a Hover Test of a 0.658-Scale V-22 Rotor and Wing," NASA TM 102244, February 1990.

2 Steger, J., Dougherty, F. C., and Benek, J., "A Chimera Grid Scheme," Advances in Grid Generation, K. N. Ghia and U. Ghia, eds., ASME FED-Vol 5., June 1983.

${ }^{3}$ Meakin, R., "A New Method for Establishing Intergrid Communication Among Systems of Overset Grids," AIAA Paper 91-1586-CP, pp. 662-671, June 1991.

${ }^{4}$ Dietz, W., Jacocks, J., and Fox, J., "Application of Domain Decomposition to the Analysis of Complex Aerodynamic Configurations," SIAM Conf. Domain Decomposition Methods, Houston, TX, March 1989.

${ }^{5}$ Brown, D., Chesshire, G., Henshaw, W., and Kreiss, O., "On Composite Overlapping Grids," 7th International Conference on Finite Element Methods in Flow Problems, Huntsville, AL, April 1989.
6 Maple, R. and Belk, D., "Automated Set Up of Blocked, Patched, and Embedded Grids in the Beggar Flow Solver," Numerical Grid Generation in Computational Fluid Dynamics and Related Fields, ed. N.P. Weatherill et al., 1994, Pine Ridge Press, pp. $305-$ 314.

7 Meakin, R., "Computations of the Unsteady Flow About a Generic Wing/Pylon/Finned-Store Configuration," AIAA Paper 92-4568-CP, pp. 564-580, August 1992.

${ }^{8}$ Meakin, R., "Moving Body Overset Grid Methods for Complete Aircraft Tiltrotor Simulations," AIAA Paper 93-3350-CP, pp. 576-588, July 1993.

${ }^{9}$ Renze, K., Buning, P., and Rajagoplan, "A Comparative Study of Turbulence Models for Overset Grids," AIAA Paper 92-0437, January 1992.

${ }^{10}$ Pulliam, T. and Steger, J., "On Implicit Finite Difference Simulations of Three-Dimensional Flow," AIAA Paper 78-10, January 1978.

11 Warming, R. and Beam, R., "On the Construction and Application of Implicit Factored Schemes for Conservation Laws," SIAM-AMS Proc., Vol. 11, pp. 85$129,1978$.

12 Baldwin, B. and Lomax, H., "Thin-Layer Approximation and Algebraic Model for Separated Turbulent Flows," AIAA Paper 78-257, January 1978.

${ }^{13}$ Meakin, R., "On the Spatial and Temporal Accuracy of Overset Grid Methods for Moving Body Problems," AIAA 94-1925-CP, pp. 858-871, June 1994.

${ }^{14}$ Meakin, R., "An Efficient Means of Adaptive Refinement Within Systems of Overset Grids," AIAA 951722-CP, June 1995.

15 Lane, D., "UFAT - A Particle Tracer for TimeDependent Flow Fields," Proceedings of IEEE Visualization '94, pp. 257-264, October 1994.

${ }^{16}$ Swanson, A. and Light, J., "Shadowgraph Flow Visualization of Isolated Tiltrotor and Rotor/Wing Wakes," 48th AHS Forum Proceedings, pp 13231344, June 1992.

17 Fejtek, I. and Roberts, L., "Navier-Stokes Computation of Wing/Rotor Interaction for a Tilt Rotor in Hover," AIAA Paper 91-0707, 29th Aerospace Sciences Meeting, Reno, January 1991. 